bioRxiv preprint doi: https://doi.org/10.1101/2021.10.29.466512. this version posted November 1.2021. The copyright holder for this preprint (which was not certified by peer review) is the author/funder, who has granted bioRxiv a license to display the preprint in perpetuity. It is made available under aCC-BY-NC-ND 4.0 International license.

1

2

3

4

5

\section{Sex and Estrous Cycle in Memory for Sequences of Events in Rats} Abbreviated Title: SEX AND ESTROUS IN SEQUENCE MEMORY

\author{
M. Jayachandran ${ }^{1}$, P. Langius ${ }^{1}$, F. Pazos Rego ${ }^{1}$, R. P. Vertes ${ }^{2}$, T. A. Allen ${ }^{1,2,3}$
}

${ }^{1}$ Cognitive Neuroscience Program, Florida International University, Miami FL, 33199

Correspondence: tallen@fiu.edu

${ }^{2}$ Center for Complex Systems and Brain Sciences, Florida Atlantic University, Boca Raton, FL 33431, USA

${ }^{3}$ Department of Environmental Health Sciences, Robert Stempel College of Public Health, Florida International University, Miami, FL 33199, USA

Number of Figures: 5

Number of tables: 0

Number of Text Pages: 31

Title: 15

Abstract: 248

Introduction: 784

Methods: 1622

Results: 2931

Discussion: 1233

Acknowledgement: 59

Author Contributions: 38

Figure Captions: 864

Total: 7780

Corresponding Author:

Timothy A. Allen, PhD

Department of Psychology

Florida International University

11200 SW $8^{\text {th }}$ Street

Miami, FL 33199

email: tallen@fiu.edu 
47 The ability to remember sequences of events is fundamental to episodic memory. While rodent studies have examined sex and estrous cycle in episodic-like spatial memory tasks, little is known about these biological variables in memory for sequences of events that depend on representations of temporal context. We investigated the role of sex and estrous cycle in rats during all training and testing stages of a cross-species validated sequence memory task (Jayachandran et al., 2019). Rats were trained on a task composed of two sequences, each with four unique odors delivered on opposite ends of a linear track. Training occurred in six successive stages starting with learning to poke in a nose port for $\geq 1.2 \mathrm{~s}$; eventually demonstrating sequence memory by holding their nose in the port for $\geq 1 \mathrm{~s}$ for in-sequence odors and $<1$ s for out-ofsequence odors in order to receive a water reward. Performance was analyzed across sex and estrous cycle (proestrus, estrus, metestrus, and diestrus), the latter being determined by the cellular composition of a daily vaginal lavage. We found no evidence of sex differences in asymptotic sequence memory performance, similar to published data in humans performing the analogous task (Reeders et al., 2021). Likewise, we found no differences in performance across the estrous cycle. One minor difference was that female rats tended to have slightly longer poke times, while males had slightly more short poke times but this did not affect their decisions. These results suggest sex and estrous cycle are not major factors in sequence memory capacities. 
73

74

75

76

77

78

79

80

81

82

83

84

85

86

87

88

89

90

91

92

93

94

95

96

97

98

99

SEX AND ESTROUS IN SEQUENCE MEMORY

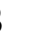

INTRODUCTION

Episodic memory is the ability to encode and recollect our daily personal experiences (Tulving, 2002;

Allen \& Fortin, 2013). A fundamental feature of episodic memory is the ability to remember sequences of

events as they occurred across time (Allen et al., 2020; Allen et al., 2014, 2016; Eichenbaum \& Fortin, 2005;

Jayachandran et al., 2019). While rodent studies have examined sex and estrous cycle as variables in

spatial and episodic-like memory tasks, little is known about these biological variables in a sequence

memory task that depends on representations of temporal contexts. The study of sex and sex hormones in

sequence memory is particularly important in understanding the neurobiology of mental health disorders

because of known sex differences and the prevalence of deficits in temporal cognition (Li \& Singh, 201;4

Postma et al., 2004; Valera et al., 2010) in disorders such as schizophrenia and Alzheimer's disease

(Kessler et al., 2005; Launer, 1999; Pigott, 2003).

Sex differences in memory have been widely reported in human and nonhuman animals alike (Levy et al., 2005; Seymoure et al., 1996; Williams et al., 1990). For example, rodent studies have shown that sex

impacts both spatial cognition and object recognition (Barha et al., 2010; Hamson et al., 2016; Koss \& Frick, 2017; Luine et al., 2017; Spritzer et al., 2008). Most commonly, evidence suggests that human and rodent males have an advantage in tests of spatial memory (Driscoll, 2005; Jonasson, 2005; Nowak et al., 2014;

Steimer \& Woolley et al., 2010). Importantly, when strategy is considered or a landmark, such as a wall cue is included, these sex differences disappear (Chai \& Jacobs, 2010; Saucier et al., 2008). Alternatively, females tend to outperform males in object memory (Bettis \& Jacobs, 2012; Levy et al., 2005; Sutcliffe et al., 2007). However, not every study finds sex differences in spatial learning and memory tasks (Bucci et al., 2021; Frick et al., 1999; Healy et al., 1999).

When looking at sex differences in memory, it is important to consider the bioavailability of key sex hormones. For example, exogenous ovarian hormones have been shown to enhance performance in object memory in in both rodent and human females (Walf \& Frye, 2006). This suggests the sex differences in memory can be dependent on the levels of circulating gonadal hormones. Important to our experiments here, hormone levels change reliably during the estrous cycle in females and different phases have been linked to differences in memory. For example, Healy and colleagues, (1999) showed that female rodents 
were slowest to locate the platform in a Morris water maze task during estrus and were fastest during

proestrus which was attributed to a core spatial memory difference. Likewise, in other spatial tasks, females

demonstrate the use of allocentric strategies during proestrus and egocentric/mixed strategies during

diestrus (Korol, 2004; Koss \& Frick, 2017). In object memory tasks, rats in proestrus outperformed rats in

studies not all studies find differences in memory across the estrous cycle. For example, Stackman et al., (1997) did not observe significant differences in acquisition or performance levels across the estrous cycle

when testing working memory using a radial arm maze; however, they showed that rats performed the task significantly slower during proestrus than on any other estrous cycle phase. This suggests that while the underlying working memory process remained stable, other aspects of performance can vary across the estrous cycle.

Here, we investigated the role of sex and estrous cycle (proestrus, estrus, metestrus, diestrus) in rats during training and testing stage of an odor sequence memory task (Jayachandran et al., 2019). Rats were trained for several months on the sequence memory task composed of two four-odor sequences delivered at opposite ends of a linear track. Training occurred progressively across six major stages starting with learning to poke and hold for $\geq 1.2 \mathrm{~s}$ for a small water reward to eventually differentiating in-sequence (InSeq) and out-of-sequence (OutSeq) odors, one at a time, in four odor sequence sets. Performance across training stages was analyzed across sex and estrous cycle phases, the latter of which was determined by the cellular composition of a vaginal lavage. We conducted an extensive analysis of behavior at each stage including trials to criterion, self-paced inter-odor interval times, self-paced inter-sequence interval times, poke times under different sequential and accuracy conditions, and overall sequence memory. We found no evidence that males and females differed in learning, remembering, or performing the sequence memory task. Moreover, we did not find any differences across the estrous cycle in sequence memory. The results suggest sex and estrous cycle are not major factors in memory for sequences of events. 
Subjects: Twenty Long-Evans rats (10 females; Charles River Laboratories; weighing 250-350g upon arrival) were used. Two male rats failed to progress past the TwoOdor stage of training and were excluded from all analysis. Rats were individually housed and maintained on a 12-hr inverse light/dark cycle (lights were turned off at $10 \mathrm{AM})$. Rats had ad libitum access to food, but access to water was limited to $3-5 \mathrm{~min}$ each day, depending on how much water they received as a reward during behavioral training $(6-9 \mathrm{~mL})$. All training sessions and lavages were conducted during the dark phase (active period) of the light cycle. All experimental procedures using animals were conducted in accordance with the Florida International University Institutional Animal Care and Use Committee (FIU IACUC).

Task Apparatus: Rats were tested in a noise-attenuated experimental room. The behavioral apparatus consisted of a linear track (length, $183 \mathrm{~cm}$; width, $10 \mathrm{~cm}$; height, $43 \mathrm{~cm}$ ) with walls angled outward at $15^{\circ}$ and nose ports centered at each end through which repeated deliveries of multiple distinct odors could be presented. Photobeam sensors were used to detect nose port entries. Each nose port was connected to an odor delivery system (Med Associates; Fairfax, VT). Odor deliveries were initiated by a nose-poke entry and terminated either when the rat withdrew their poke or after $1 \mathrm{~s}$ had elapsed. Water ports were positioned under each nose port for reward delivery. Timing boards (Plexon; Dallas, TX) and digital input/output devices (National Instruments; Austin, TX) were used to measure all event times and control the hardware. All aspects of the task were automated using custom MATLAB scripts (MathWorks 2021a; Natick, MA). A 256-channel OmniPlex with video tracking and CinePlex behavioral software (Plexon; Dallas, TX) were used to interface with the hardware in real time and record behavioral data. Odors were organic odorants contained in glass jars (Sigma-Aldrich; St. Louis, MO, A1: 1-octanol, CAS: 111-87-5; B1: (-) - limonene, CAS: 5989-54-8; C1: I-menthone, CAS: 14073-97-3; D1: isobutyl alcohol, CAS: 78-83-1; A2: acetophenone, CAS: 98-86-2; B2: (1S) - (-) - beta-pinene, CAS: 18172-67-3; C2: L (-) - carvone, CAS: 6485-40-1; D2: 5methyl-2-hexanone, CAS: 110-12-3) that were volatilized with nitrogen air (flow rate, 2L/min) and diluted with ultrapure air (flow rate, $1 \mathrm{~L} / \mathrm{min}$ ). To prevent cross-contamination, separate Teflon tubing lines were used for each odor. These lines converged into a single channel at the bottom of the odor port. In addition, a 
vacuum located at the top of the odor port provided constant negative pressure to quickly evacuate odor traces with a matched flow rate.

Sequence Memory Task: The sequence memory task (Jayachandran et al., 2019; Allen et al., 2014) involves repeated presentations of odor sequences and requires the rat to determine whether each item (odor) presented is in-sequence (InSeq) or out-of-sequence (OutSeq). Rats were trained on two sequences, each comprised of four distinct odors (e.g., Seq1: $A_{1} B_{1} C_{1} D_{1}$, Seq2: $A_{2} B_{2} C_{2} D_{2}$ ). Each sequence was presented at either end of a linear track maze. Odor presentations were initiated by a nose-poke, and each trial was terminated after the rat either held the nose-poke response for $\geq 1 \mathrm{~s}$ (InSeq) or withdrew its nose-

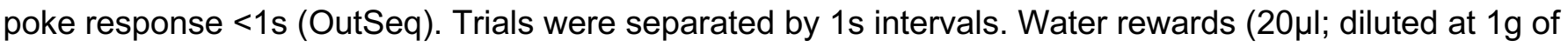
aspartame for every $500 \mathrm{~mL}$ of water) were delivered below the odor port after each correct response. Incorrect responses triggered a buzzer sound and the termination of the sequence. Each sequence was presented 50-100 times per session; approximately half the presentations included all items InSeq (ABCD) and half included one item OutSeq (e.g., ABADD, odor A repeated in the 3rd position). Note that OutSeq items could be presented in any sequence position except the first position (i.e., sequences always began with an InSeq item). Sequence memory was probed with OutSeq trials (e.g., ABAD; one OutSeq trial randomly presented per sequence).

Sequence Memory Task Training: Naive rats were initially trained on a series of incremental stages over 20-30 weeks. Each rat was trained to poke and hold its nose in an odor port to receive a small water reward. The minimum required nose-poke duration started at $50 \mathrm{~ms}$ and was gradually increased (in $15 \mathrm{~ms}$ increments) until the rat held the nose-poke position for $\geq 1.2 \mathrm{~s} \sim 75 \%$ of the time over three sessions $(75-100$ nose-pokes per session). The rats were then trained to poke on side 2 until reaching criterion; poking for $\geq 1.2$ s with $\sim 75 \%$ accuracy for three consecutive sessions. The rats were then habituated to odor presentations in the port (odor $A_{1}$ and $A_{2}$ ). The hold time was reduced down to $1 \mathrm{~s}$ and rats were required to alternate between sides for a small water reward at each side for $\sim 75 \%$ accuracy for three consecutive sessions before moving on. Then a second set of odors was introduced (Seq1: $A_{1} B_{1}$ and Seq2: $\left.A_{2} B_{2}\right)$ and 
79

80

81

82

83

84

85

86

87

88

89

90

91

92

93

94

95

96

97

98

99

00

01

02

03

04

05

each rat was required to maintain its nose-poke response for $\geq 1 \mathrm{~s}$ to receive a reward and needed to achieve $\sim 70 \%$ accuracy before moving on. The rats were next trained to identify InSeq and OutSeq items.

Rats began this stage trained on a two-item sequence: they were presented with "AB" and "A $\underline{A}$ "

sequences. The correct response to the first odor was to hold their nose-poke for $\geq 1$ s (Odor $A$ always being the first item). For the second odor, rats were required to determine whether the item was InSeq (AB; hold

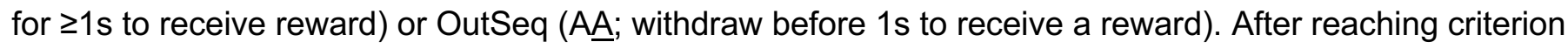
on the two-item sequence, the number of items per sequence was increased to three odors each side (Seq1: $A_{1} B_{1} C_{1}$ and Seq2: $A_{2} B_{2} C_{2}$ ), and four odors each side (Seq1: $A_{1} B_{1} C_{1} D_{1}$ and Seq2: $A_{2} B_{2} C_{2} D_{2}$ ) in successive stages. The criterion for these stages set percentages as indicated in the results or the ability differentiate InSeq and OutSeq items above a sequence memory index (SMI; see quantitative and statistical analysis) of 0.2 over three consecutive sessions. Once rats reached criterion for the last stage of training, 15 consecutive sessions were collected to examine both sex and estrous cycle differences in overall SMI.

Lavaging: Phases of the estrus cycle were determined in each female rat using vaginal lavage within four hours of each behavioral session. Rats were restrained using a designated towel and a drop of sterilized saline $(\mathrm{NaCl}, 0.85 \%)$ was placed over the vaginal opening to clean the area. The tip of a sterilized transfer pipette filled with sterilized saline $(\sim 0.25-0.5 \mathrm{~mL})$ was inserted into the vaginal opening to extract vaginal cells. All vaginal smears were placed on glass slides and photomicrographs were taken under brightfield illumination using an Olympus BX41 following guidelines from Westwood (2008). To control for experimenter handling, male rats were handled similarly and poked around the anal opening with a clean transfer pipette.

Estrous Cycle Cytology: Estrous cycles in rats typically last four to five days and have four distinct phases: proestrus, estrus, metestrus and diestrus; each phase corresponds with specific cell types, i.e., nucleated epithelial cells, amorphous cornified cells, and small round leukocytes. Proestrus is characterized predominantly by nucleated epithelial cells, estrus predominantly amorphous cornified cells, metestrus by a mixture of nucleated epithelial cells, cornified cells and leukocytes, and diestrus predominantly leukocytes. 
All vaginal cell samples were obtained and imaged, then the images were blindly classified by three investigators.

\section{Quantification and Statistical Analysis:}

All data was analyzed in MATLAB 2021a (MathWorks; Natick, MA), SPSS 20.0.0, and Excel 2016 using custom scripts and functions. Performance on the task can be analyzed using a number of measures (Allen et al., 2014). The first position of each sequence was excluded from all analysis as these items are always InSeq. Expected vs. observed frequencies were analyzed with $\mathrm{G}$ tests to determine whether the observed frequencies of InSeq and OutSeq responses for a given session were significantly different from the frequency expected by chance. $\mathrm{G}$ tests provide a measure of performance that controls for response bias and is a robust alternative to the $x^{2}$ test, especially for datasets that include cells with smaller frequencies (Sokal \& Rohlf, 1995). To compare performance across sessions or animals, a SMI was calculated (Allen et al., 2014) as shown in the following equation:

$$
S M I=\frac{\left(0.9 * I N_{o u t}\right)\left(0.1 * O U T_{c o r}\right)-\left(0.9 * I N_{i c c}\right)\left(0.1 * O U T_{i c c}\right)}{\sqrt{\left(0.9 * I N_{c o r}+0.9 * I N_{i n c}\right)\left(0.1 * O U T_{c o r}+0.1 * O U T_{i c c}\right) *\left(0.9 * I N_{c o r}+0.1 * O U T_{i c c}\right)\left(0.9 * I N_{\text {inc }}+0.1 * O U T_{c o r}\right)}}
$$

The SMI normalizes the proportion of InSeq and OutSeq items presented during a session and reduces sequence memory performance to a single value ranging from -1 to 1 . A score of 1 represents a perfect sequence memory performance in which a subject would have correctly held their nose-poke response to all InSeq items and correctly withdrawn on all OutSeq items. A score of 0 indicates chance performance.

Negative SMI scores represent performance levels below that expected by chance. SMI was calculated for males and females as well as between estrous phases during the two, three, and four odor stages of the sequence task. Using SPSS, an independent t-test was used to determine if males and females differed in their overall SMI. We went on to examine the differences between sequence 1 and sequence 2 within sex and estrous cycle by running a two-way ANOVA. A one-way ANOVA was used to determine if overall SMI differed within female estrous cycle. Linear regressions were performed in increments of pre-determined set trials or sessions to compare males and females in order to determine learning at each stage of training on the sequence task. Independent sample t-tests were used to analyze whether males and females differed in 
self-paced inter-trial-interval (ITI), inter-odor-interval (IOI), and inter-sequence interval (ISI). Nose-poke duration was analyzed using independent t-tests to determine whether rats held their responses significantly longer in InSeq $_{\text {correct }}$ than in OutSeq $q_{c o r r e c t}$ trials. General poke distributions were created through MATLAB using the session data.

\section{Overall Sequence Memory Task and Training}

We trained male $(n=10)$ and female $(n=10)$ rats in an odor sequence memory task (Jayachandran et al., 2019). The behavioral setup was automated and allowed repeated delivery of pure chemical odorants between two odor ports located at opposite ends of a linear track (Figure1A). The rats were trained to indicate if an odor is InSeq by holding their nose in the nose-port for $\geq 1$ s (Figure 1Bi) or OutSeq by withdrawing their nose prior to $1 \mathrm{~s}$ for a small water reward (Figure 1Bii). Rats were trained in progressive stages over several months on the sequence task, as depicted in Figure 1C. At each stage of training on the sequence task, rats had to reach a specific performance criterion in order to progress to the next stage. Once they reached the final stage (FourOdors) and behavioral criterion, we collected 15 consecutive sessions to analyze overall sequence memory. Two male rats were not able to continue past TwoOdors and therefore we removed them from the overall analysis. We calculated an SMI to measure overall sequence memory while controlling for individual differences in poke-hold behavior (Allen et al., 2014). We averaged

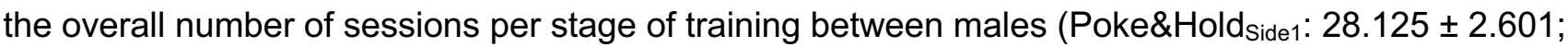
${\text { Poke } \& H_{\text {old }}}_{\text {side2 }}: 8.625 \pm 1.731$; OneOdor: $15.500 \pm 2.706$; TwoOdors: $49.375 \pm 8.268$; ThreeOdors: 33.500 \pm 8.203; FourOdors: $30.625 \pm 1.499$ ) and females (Poke\&Hold ${ }_{\text {side } 1:} 22.800 \pm 1.504 ;$ Poke\&Hold side2 $_{2} 8.400 \pm$ 1.962; OneOdor: $13.300 \pm 1.506$; TwoOdors: $40.600 \pm 4.861$; ThreeOdors: $30.800 \pm 3.782$; FourOdors: $30.400 \pm 0.957)$. There were no significant differences in the number of sessions to criterion for stage when comparing males and females $\left(\right.$ Poke\&Hold ${ }_{\text {Side1 }}: \mathrm{t}_{(16)}=-1.861, p=0.081 ;$ Poke\&Hold $_{\text {Side2: }}: \mathrm{t}_{(16)}=-0.084, p=$ 0.934; OneOdor: $\mathrm{t}_{(16)}=-0.749, p=0.465 ;$ TwoOdors: $\mathrm{t}_{(16)}=-0.959, p=0.352 ;$ ThreeOdors: $\mathrm{t}_{(16)}=-0.320, p=$ 0.753; FourOdors; $\left.\mathrm{t}_{(16)}=-0.131, p=0.897\right)$. 
60

61

62

63

64

65

66

67

Rats were first trained to poke and hold in the nose port located on side 1 of the sequence memory task (Figure 2Ai). During this stage, the rat is trained to hold their nose in the nose port with a start hold time of $50 \mathrm{~ms}$. With each correct trial, the hold time was increased by $15 \mathrm{~ms}$ until reaching a $1.2 \mathrm{~s}$ hold time. The rats were then required to poke for $\geq 1.2 \mathrm{~s}$ at $\sim 75 \%$ accuracy for 3 consecutive sessions. We first examined the learning rates of males and females by calculating the slope over 200 trial increments (Figure 2Aii). Males and females did not show any significant differences in learning during Poke\&Hold $\mathrm{side1}_{\text {(Trials }}$ (-200: $_{\mathrm{t}_{(16)}}=$ 1.075, $p=0.299 ;$ Trials $\mathrm{s}_{200-400}: \mathrm{t}_{(16)}=0.850, p=0.262 ;$ Trials $_{400-600:} \mathrm{t}_{(16)}=0.336, p=0.741 ;$ Trials $_{600-800}: \mathrm{t}_{(16)}=$ $0.840, p=0.413$; Trials $\left._{800-1000:} \mathrm{t}_{(16)}=0.754, p=0.462\right)$. A few rats exceeded 1000 trials to progress to the next stage of the task; however, due to too few values we could not calculate an average past 1000 trials. We then looked at the poke distributions between males and females for all trials. Overall, the proportion of nosepokes remained similar between males and females with a slight increase for males on short pokes (Figure 2Aiii). We performed several analyses to test the alternative hypothesis that non-memory-related behavioral effects account for impaired performance in the sequence task. We measured the time it took rats to initiate each poke (inter-trial-interval; ITI). The ITI did not differ significantly between males and females (Figure 2Aiv; $\mathrm{t}_{(410)}=-0.174, p=0.862$ ).

Once reaching criterion for Poke\&Hold $\mathrm{side}_{1}$ rats were then trained on Poke\&Hold $\mathrm{dide2}_{2}$ where rats were familiarized with the odor port on side 2 and trained to poke for $\geq 1.2 \mathrm{~s}$ at $\sim 75 \%$ accuracy for 3 consecutive sessions (Figure 2Bi). We calculated the proportion correct for each session after poke hold times became consistent. We examined learning between males and females by measuring proportion correct and calculating the rate of change in proportion correct (Figure 2Bii; 5 session increments). A few rats continued past 10 sessions; however we were not able to calculate an average due to the low sample sizes after this point. Males and females did not show any significant differences in learning $\left(\right.$ Sessions $_{1-5}: \mathrm{t}_{(16)}=-0.545, p=$ 0.593; Sessions $\left.5-10: t_{(7)}=-0.124, p=0.905\right)$. When we looked at poke distributions, the proportion of nosepokes between males and females remained similar (Figure 2Biii). However, we do note that males seem to have more short pokes $(\sim 0.1 \mathrm{~s})$ while females tended to hold longer $(\sim 1.4 \mathrm{~s})$. We then examined ITI and found no significant differences between males and females (Figure 2Biv; $\mathrm{t}_{(142)}=-0.258, p=0.797$ ). 
presentation on either end of the linear track. The hold time criterion was also reduced down to $1 \mathrm{~s}$ and rats

were required to alternate between sides (Figure $2 \mathrm{Ci}$ ). Rats were required to reach $\sim 75 \%$ accuracy for 3

consecutive sessions at this stage. We found no significant differences in learning between males and

females (Sessions ${ }_{1-5}: \mathrm{t}_{(16)}=-1.123, p=0.278$; Sessions ${ }_{5-10}: \mathrm{t}_{(16)}=1.034, p=0.317$; Sessions $_{10-15}: \mathrm{t}_{(7)}=0.774$,

$p=0.464)$. We then looked at poke distributions between males and females; overall, we found they were

very similar. Again, we observed males' tendencies towards short pokes ( $0.1-0.2 s)$ with females'

tendencies towards longer pokes $(\sim 1.2-1.5 \mathrm{~s})$. At this stage we measured the time it took the rats to run

between sequences which we refer to as inter-sequence-interval (ISI). We found no significant differences in

ISI between males and females (Figure 2Civ; $\mathrm{t}_{(224)}=0.655, p=0.513$ ). Overall, these results suggest that

males and females show little to no differences in their poking and timing behaviors.

\section{Males and Females Perform Similarly on the Sequence Memory Task}

After reaching criterion for the OneOdor stage of training, rats are then moved onto TwoOdors where

they are trained to poke for two separate odors on each side (Figure 3Ai; Seq1: $A_{1} B_{1}$ and Seq2: $A_{2} B_{2}$ ). Once rats are able to poke twice on each side with $\sim 70 \%$ accuracy, they are then trained to identify InSeq and trained until they demonstrated sufficient performance ( $\geq 0.2 \mathrm{SMI})$ for three consecutive sessions. We 
0.938, $p=0.363$; Sessions $\left.{ }_{15-20}: \mathrm{t}_{(10)}=-0.394, p=0.702\right)$. We also examined the overall SMI of the 3 consecutive stages in which criterion was met. We found no significant difference between the sexes (Figure 3Aiii; $\mathrm{t}_{(52)}=1.134, p=0.262$, Male: $0.347 \pm 0.022$, Female: $0.386 \pm 0.026$ ). Further, we examined whether there were differences between sequence 1 and sequence 2. A two-way ANOVA revealed no statistically significant interaction between sex and sequence $\left(F_{(3,104)}=0.031, p=0.860\right)$. Simple main effect analysis showed that sex and sequence did not have a statistically significant effect on SMI (Sex: $p=0.304$; Sequence: $p=0.643)$.

We examined the nose-poke distributions for both InSeq and OutSeq trials (Figures 3Av and 3Avi). The InSeq distribution showed the proportion of nose-pokes in males and females remained similar. On OutSeq trials, however, we see a modest difference between male and female poke times. Females tended to poke for OutSeq trials close to the $1 \mathrm{~s}$ threshold $(\sim 0.8-0.99 \mathrm{~s})$ whereas males continued to show short pokes $(\sim 0.1-0.4 s)$ in both InSeq and OutSeq trials. We evaluated whether males and females differed in nosepoke times on InSeq $\mathrm{q}_{\mathrm{correct}}$ and OutSeq $\mathrm{q}_{\mathrm{corect}}$ trials (Figure 3Avii). No significant differences were detected between males and females for $\operatorname{InSeq}_{\text {correct }}$ trials $\left(\mathrm{t}_{(373)}=1.195 p=0.233\right)$. However, in OutSeqcorrect trials we found a significant difference, with females showing a slightly longer hold time $\left(\mathrm{t}_{(373)}=9.609, p=1.119 \times 10^{-}\right.$ 19; Male: $0.446 \pm 0.020$; Female: $0.678 \pm 0.014$ ) which corresponds with what we observed in the poke distribution. We then looked at the time rats spent between each odor trial (inter-odor-interval; IOI) and ISI. The IOI did not differ significantly between males and females (Figure 3Aviii; $\mathrm{t}_{(767)}=1.495, p=0.135$ ), suggesting rats collected water rewards and engaged with the odors at similar rates. Furthermore, we found no effect by group on the ISI (Figure $\left.3 \mathrm{Aix} ; \mathrm{t}_{(757)}=0.312, p=0.755\right)$, suggesting rats in both groups alternated at similar rates between sequences.

Once rats reach criterion in the TwoOdor training stage, they are moved to ThreeOdors. In this stage, one more odor is added to each sequence (Figure 3Bi; Seq1: $A_{1} B_{1} C_{1}$ and Seq2: $A_{2} B_{2} C_{2}$ ). Rats were trained to poke three times on either side with at least $\sim 65 \%$ accuracy before being introduced to the OutSeq probe trials. With this three-item sequence the number of possible OutSeq configurations has expanded, making this the first stage to test sequence memory. Rats were trained up until they demonstrated sufficient sequence memory ( $\geq 0.2 \mathrm{SMI}$ ) for three consecutive trials to move onto the final training stage. We 
averaged the first twenty sessions to measure learning. We found a significant difference in the first five sessions where males seemed to learn at a faster rate compared to females (Figure $3 \mathrm{Bii}$; Sessions ${ }_{1-5}: \mathrm{t}_{(16)}=$ $-2.471, p=0.025)$. However, this difference diminished with the subsequent sessions (Sessions ${ }_{5-10}: \mathrm{t}_{(14)}=$ 1.131, $p=0.277$; Sessions ${ }_{10-15}: \mathrm{t}_{(12)}=-0.037, p=0.971$; Sessions $\left.{ }_{15-20}: \mathrm{t}_{(9)}=-0.206, p=0.842\right)$.

We then examined the overall SMI using data from after subjects had reached the behavioral criterion. We found no significant differences between males and females (Figure 3Biii; $\mathrm{t}_{(52)}=-1.755, p=0.085$, Male: $0.428 \pm 0.015$, Female: $0.394 \pm 0.012$ ). We also observed no significant interactions between sex and sequence $\left(F_{(3,104)}=0.059, p=0.808\right)$. A simple main effect analysis showed that sex and sequence did not have a statistically significant effect on SMI (Sex: $p=0.094$; Sequence: $p=0.182$ ).

We then evaluated the nose-poke distributions for both InSeq and OutSeq trials (Figure 3Bv and 3Bvi). The InSeq distribution shows a similar proportion of nose-pokes in males and females. The OutSeq trials showed that males poked between $\sim 0.4-0.7 \mathrm{~s}$ whereas females tended to poke between $\sim 0.8-0.95 \mathrm{~s}$. We also saw more short pokes from males in both InSeq and OutSeq trials. We evaluated non-mnemonic behaviors by examining nose-poke behaviors, IOI, and ISI. Additionally, InSeq correct $_{\text {nose-poke times }}$ between males and females did not significantly differ (Figure 3Bvii: $\mathrm{t}_{(344)}=1.718, p=0.087$ ). There was a

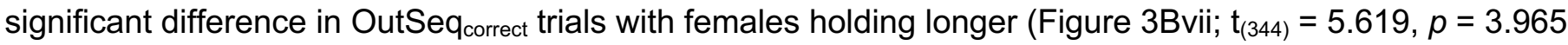
x 10-8; Males: $0.501 \pm 0.021$; Females: $0.643 \pm 0.015)$. The IOI and ISI did not differ significantly between males and females (Figure 3Bviii and 3Bix; IOI: $\mathrm{t}_{(562)}=1.568, p=0.118$; ISI: $\mathrm{t}_{(562)}=1.431, p=0.153$ ). Overall, these results suggest that males and females do not differ in working memory. However, males show a slight advantage in learning the OutSeq rule during the ThreeOdor training stage.

Finally, upon successfully completing the ThreeOdor training stage, an additional odor is introduced to each sequence (Seq1: $A_{1} B_{1} C_{1} D_{1}$ and Seq2: $A_{2} B_{2} C_{2} D_{2}$ ). Rats were trained until they achieved $\sim 65 \%$ accuracy in differentiating between InSeq and OutSeq ( $\geq 0.2 \mathrm{SMI})$. Once this criterion was met, we then ran the rats for 15 consecutive sessions (Figure 4). We took the averaged results from 25 sessions after the task was learned in order to describe overall learning and performance. We found no significant difference in learning between males and females. (Figure 4B; Sessions ${ }_{1-5}: t_{(16)}=0.221, p=0.828$; Sessions $5-10: t_{(16)}=-$ 0.176, $p=0.862$; Sessions ${ }_{10-15}: \mathrm{t}_{(16)}=-0.214, p=0.833 ;$ Sessions $_{15-20}: \mathrm{t}_{(16)}=-0.437, p=0.668$; Sessions $20-25$ : 
$\left.t_{(14)}=1.701, p=0.111\right)$. By session ten, most rats reached behavioral criterion (asymptotic sequence memory performance levels over multiple sessions). Overall, rats demonstrated strong sequence memory (Figure 4C; Male: $0.262 \pm 8.690 \times 10^{-3}$, Female: $0.272 \pm 8.120 \times 10^{-3}$ ) and performance did not differ significantly between males and females (Figure $4 \mathrm{Ci}$; SMI: $\mathrm{t}_{(268)}=0.784, p=0.434$ ). Both males and females performed well above chance levels on sequence 1 (Male: $0.268 \pm 0.017$; Female: $0.280 \pm 0.018$ ) and sequence 2 (Male: $0.279 \pm 0.016$; Female: $0.318 \pm 0.018$ ), with no significant interactions between sequences or sex (Figure 4Cii; $F_{(1,479)}=0.608, p=0.436$ ). Simple main effect analysis showed that sex and sequence did not have a statistically significant effect on SMI (Sex: $p=0.160$; Sequence: $p=0.177$ ), indicating rats successfully switched between the two sequences.

We evaluated non-mnemonic effect of sex by examining IOI and ISI. The IOI and ISI did not differ significantly between males and females (Figure 4D and 4E; IOI: $\mathrm{t}_{(278)}=-0.569, p=0.570 ; I_{\text {ISI: }} \mathrm{t}_{(278)}=0.162$, $p=0.871)$. We analyzed the poke distribution between males and females (Figure 4Fi and Fii). The InSeq and OutSeq distribution showed the proportion of nose-pokes remained similar between males and females. However, there was a slight increase in the proportion of InSeq and OutSeq nose-pokes near the short distribution peak for males $(\sim 0.1-0.2 s)$. We also examined whether sex affected nose-poke times on InSeq $q_{c o r r e c t}$ and OutSeq $q_{c o r r e c t}$ trials (Figure 4G). No significant differences were detected in InSeqcorrect trials $\left(\mathrm{t}_{(268)}=-1.925, p=0.055\right)$. In the OutSeq $q_{c o r r e c t}$ trials there was a significant increase in nose-poke time for females $\left(\mathrm{t}_{(268)}=3.617, p=3.560 \times 10^{-4}\right.$; Male: $0.443 \pm 0.020$; Female: $\left.0.544 \pm 0.019\right)$. Overall, these results demonstrate that males and females do not differ in their overall sequence memory. However, there are slight differences in their hold times for OutSeq trials, which may indicate uncertainty regarding whether a trial was InSeq or OutSeq rather than a deficit related to basic nose-poke behavior or sequence memory.

\section{Estrous Cycle in Sequence Memory}

Generally, the results demonstrate that males and females do not differ in learning and performance on the sequence memory task. We then looked at estrous cycle phases within females, as the fluctuations of female hormone levels are important factors to consider when working with female animals. Figure $5 \mathrm{~A}$ illustrates the hormone level in female rats during each of the four phases of the estrous cycle. In the 
93

94

95

96

97

98

99

00

01

02

03

04

05

06

07

08

09

10

11

12

13

14

15

16

17

18

19

proestrus phase, ovulation begins with the sharp increase and decrease of progesterone, increase of

estradiol, and decrease of luteinizing hormone (LH). In the estrus phase, known as the "heat phase",

progesterone remains steady, estradiol peaks and begins to decrease while LH decreases. In the metestrus

phase, all hormones remain steady. Finally, in the diestrus phase LH begins to increase slowly.

We first examined the overall SMI once rats reach asymptotic levels on the fully learned sequence memory task. We found no significant differences between the estrous cycle phases (Figure $5 \mathrm{Bi} ; \mathrm{F}_{(3,146)}=$ 1.405, $p=0.244)$. We also observed no significant differences between estrous and sequence (Figure 5Bii: $\left.F_{(7,270)}=3.56, p=0.078\right)$. Simple main effect analysis showed that estrous and sequence were not statistically significant (Estrous: $p=0.433$; Sequence: $p=0.322$ ). We then observed the general poke distributions for InSeq and OutSeq trials between the estrous cycle phases. For InSeq trials, all estrous cycle phases showcased a cluster of pokes slightly after the $1 \mathrm{~s}$ decision threshold (Figure 5Ci). For OutSeq trials, there are two main peaks of nose-pokes around $0.2 \mathrm{~s}$ and $0.8 \mathrm{~s}$ (Figure $5 \mathrm{Cii}$ ). We did observe that there was a slight increase in poke times during estrus $(\sim 0.2-0.3 \mathrm{~s})$ in comparison to the other three phases. Next, we evaluated the nose-poke times for InSeq and OutSeq correct trials. There were no significant differences in InSeq ${ }_{c o r r e c t}$ trials between the estrous cycle phases (Figure 5D; $F_{(3,149)}=0.667, p=$ 0.573). There was a significant difference in OutSeq $q_{c o r r e c t}$ trials (Figure 5D; $F_{(3,149)}=3.301, p=0.022$ ). Post hoc comparisons using Bonferroni test revealed a significant difference between estrus and metestrus $(p=$ 0.033 ) with a mean difference poke time of $0.177 \mathrm{~s}$. We explored non-mnemonic effects relevant to the sequence task. We looked at IOI and ISI and found no significant differences between the estrous cycle phases within those conditions (Figure 5E and 5F: IOI: $\mathrm{F}_{(3,149)}=0.676, p=0.568$; ISI: $\mathrm{F}_{(3,149)}=0.416, p=$ 0.742). These results that the estrous cycle does not strongly affect overall sequence memory performance.

\section{DISCUSSION}

\section{Summary of Main Findings}

We evaluated the role of sex in rats during training and testing phases of a sequence memory task. Although this task has been used in previous studies (Allen et al., 2014, 2016a; Jayachandran et al., 2019). here we describe the stages of training and its performance criterion for the first time. Overall, both male 
and female rats learned the sequence memory task at relatively the same rate. However, there were a few differences between males and females. Males tended to have a modest number of shorter poke times compared to females, which was more evident once OutSeq trials were introduced, but this did not affect overall accuracy. Moreover, males seemed to learn at a somewhat faster rate once the sequence was expanded to include more than one OutSeq position (ThreeOdor) but this effect did not extend to the full sequences. In general, there were no noticeable sex differences in sequence memory once the task was fully learned. This finding alone, however, did not address whether the lack of sex differences could have been confounded by female rats' estrous cycle. Therefore, we looked at estrous cycle specifically and found that regardless of phase, females performed similarly well on the sequence memory task.

\section{No Evidence that Sequence Memory Differs in Males and Females}

To investigate sex differences in sequence memory, we examined males and females at each training stage of the sequence task. One main difference we observed was with the introduction of the three-item sequence. At this stage, we found males had a faster learning curve compared to females. This advantage could be due to males' tendency for short pokes, allowing them more opportunities to have chance correct responses for OutSeq trials and thus providing a modest boost in discovering the rules. This may not reflect sex differences in learning per se, but rather sex differences in strategies (Gruene et al., 2015). This has been suggested before for spatial learning paradigms, in which male rats outperform females by using a more direct strategy (McCarthy \& Konkle, 2005). This difference could also be due to Type I family-wise error. As multiple tests were run at multiple stages and therefore these differences could be minor.

Moreover, this advantage was not observed once the rats moved to the final stage of training (FourOdors), where overall sequence memory did not differ between sexes. This is further supported by Reeders et al., (2021) who did not observe sex differences in humans performing an analogous task. Importantly, sequence memory effects were consistent across two different sequences. This eliminated the possibility that males and females show preference for a specific sequence or odors throughout the entirety of a session.

Apart from examining the general learning rates at each stage, we also performed several analyses to test whether males and females differed in non-memory related behaviors in the sequence task. We saw no effects between males and females on reward retrieval activity (ITI \& IOI), on the time it took to run between 
sequence (ISI), or on the overall frequency of nose pokes in which rats held the nose poke response for $\geq 1$ s (InSeq trials). We did observe females had modestly longer hold times compared to males for OutSeq trials. This was more clearly revealed in the analysis of poke time histogram where males showed a tendency to have shorter poke times whereas females showed a tendency to have longer poke times.

Although, several studies have shown a robust male advantage in working and reference memory (Roof et al., 1993; Veng et al., 2003), other studies indicate no differences or a female advantage (Bucci et al., 2021; Healy et al., 1999; Lamberty \& Gower, 1988). These confounding observations in sex differences have been associated with different factors within the task parameters. For example, a consistent start position for each trial has been associated with a lack of sex differences (Roof \& Stein, 1999). Pre-training has also been shown to reduce sex differences (Jonasson, 2005a; Perrot-Sinal et al., 1996). Generally speaking, male and female rodents typically reach identical levels of performance on most cognitive tasks although their rates and strategies of learning may differ.

\section{No Evidence Sequence Memory Differs Across Estrous Cycle}

Several studies have postulated that hormone fluctuations across the estrus cycle may account for sex differences (McEwen \& Milner, 2017; Sherry \& Hampson, 1997; Warren \& Juraska, 1997). However, no clear consensus has emerged regarding the influence of estrous cycle on learning and memory. It has been argued that estrous cycle cannot be causally linked to more variability in females relative to males (Prendergast et al., 2014). Pompili et al. (2010) observed no variation in performance associated with cycle phase when using the radial-arm maze. Stackman et al. (1997), using a delayed nonmatching-to-sample paradigm in the radial-arm maze, found no effect of estrous cycle. Nevertheless, it is important to consider sex hormones in behavior. Therefore, we examined sequence memory across phases of estrous cycle. In general, we did not find differences between the phases of estrous cycle in sequence memory. It is possible that pre-training might have reduced the sensitivity of this assessment (Bannerman et al., 1995; D. Saucier \& Cain, 1995). However, effects of the cycle on memory are somewhat subtle and inconsistent, so they may wash out when averaging across multiple females.

Interestingly, while sequence memory was not affected by the day of estrous cycle, performance on estrus was characterized by longer OutSeq poke times. One natural issue presented by measuring estrous 
cycle for this task is the asymmetry of the length of stages; because each stage averages a different length

of time, we would expect to see far more trials occurring during these longer stages than the shorter ones.

To the extent that proestrus, metestrus and diestrus is over-represented compared to estrus, which could

have skewed the group mean. Several studies report that spatial memory tested in the Morris water maze or object placement tasks was enhanced during proestrus relative to estrus and/or diestrus in mice and rats (Frick \& Berger-Sweeney, 2001; Frye, 1995; Paris \& Frye, 2008; Pompili et al., 2010). However, the reported proestrus advantage in spatial tasks is inconsistent with other data in rats showing enhanced spatial reference memory during estrus relative to proestrus (Frye, 1995; Sutcliffe et al., 2007a; Warren \& Juraska, 1997) or no detectable effect of the cycle on memory (Berry et al., 1997; Bucci et al., 2008;

Cimadevilla et al., 2000; Conrad et al., 2004; Pompili et al., 2010; Stackman et al., 1997). While the exact cognitive bases are not yet known, Korol \& Kolo (2002) have suggested that the direction of estrogen effects on performance depends upon the availability of strategies or solutions that match the participation of neural or cognitive systems. Thus, the general effects of estrogen on memory are somewhat ambiguous (Dohanich, 2002). The sequence task used here is known to involve multiple strategies including temporal contexts, ordinal representations and working memory (e.g., Reeders et al, 2021; Jayachandran et al., 2019)

\section{Conclusions}

We present evidence that sex and estrous cycle do not influence memory for sequences of events suggesting that this core aspect of episodic memory does not differ between the sexes. These data are consistent with few other studies that have investigated sex differences and estrous cycle as it pertains to behavior in rodents as well as in humans (Bucci et al., 2021; Healy et al., 1999; Reeders et al., 2021; Schmidt et al., 2009; Stackman et al., 1997). However, further studies are needed to fully characterize the effects of sex hormones on sequence memory in both male and female rats by comparing the effects of gonadectomy and possible hormone replacement. For now, the consideration of sex and estrous cycle as a biological variable will have significantly advanced our understanding of the basic mechanisms of learning and memory. 
02 This work was supported by NIH grants R01 MH113626 and F99 NS119001. A special thanks to all the 03 members of the Allen Lab, specifically our undergraduate research assistants, Andy Garcia, Sofia Levya, 04 and Chiara Pavon, who helped with task training and data collection. Also, we would like to thank the Animal 05 Care Facility and Dr. H. Vinerean DVM, DACLAM.

06

\section{AUTHOR CONTRIBUTIONS}

08

09

10 11 12 13 14 15 16 17 18 19 20 21 22 23 24 25 26 


\section{FIGURE CAPTIONS}

A
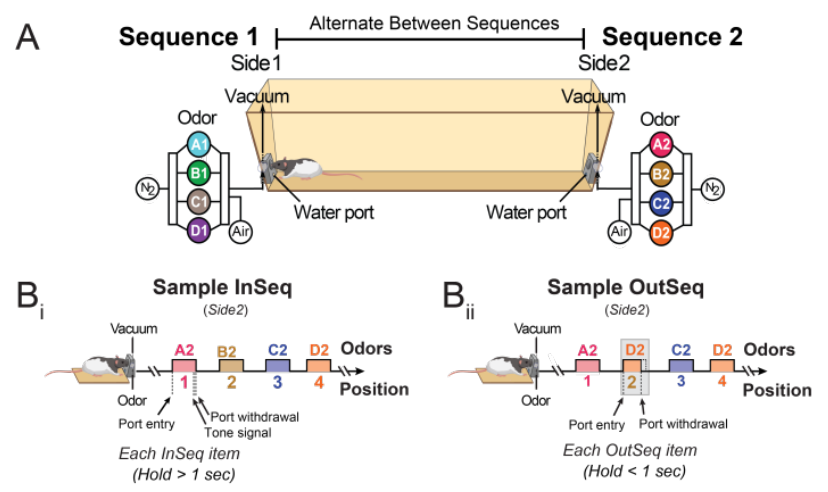

C

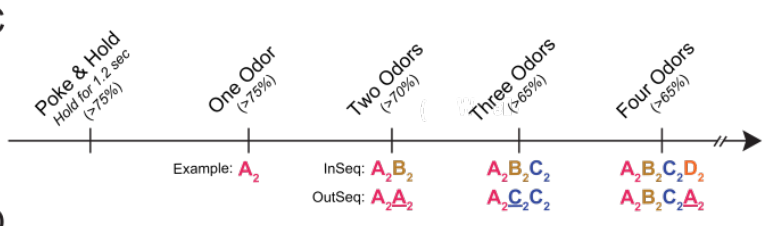

D

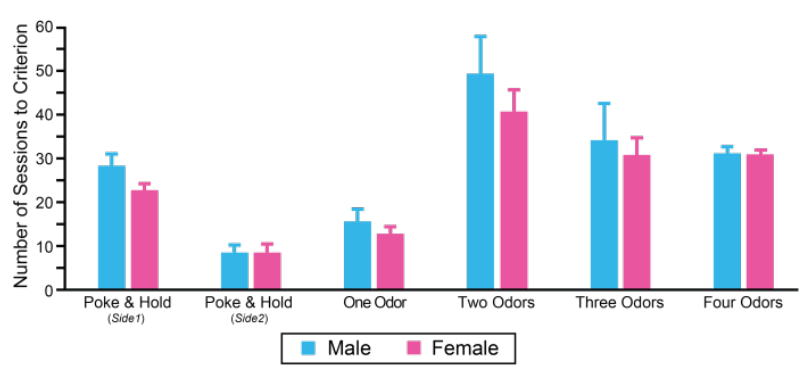

Figure 1. Sequence Memory Task

(A) A $2 \mathrm{~m}$ linear track was used with odor ports located at opposite ends where two four-odor sequences were presented (Seq1: $A_{1} B_{1} C_{1} D_{1}$ and Seq2: $A_{2} B_{2} C_{2} D_{2}$ ).

(B) Rats had to correctly identify the odors as either InSeq by holding their nose in the port for $>1 \mathrm{~s}(\mathrm{Bi})$ or OutSeq where the rat withdrew their nose prior to $1 \mathrm{~s} \mathrm{(Bii).}$

(C) Timeline showing the criterion for each training stage for the sequence memory task.

(D)Males and females did not differ in the average number of sessions per training stage.

All data are represented as mean \pm SEM. ${ }^{*} p<0.05 ;{ }^{* *} p<0.01 ;{ }^{* * *} p<0.001$ 
$A_{i}$

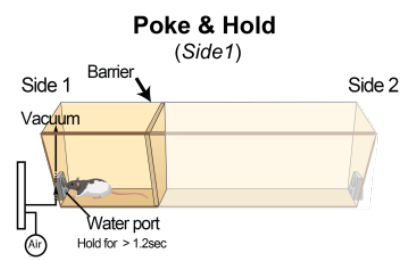

$A_{\text {ii }}$

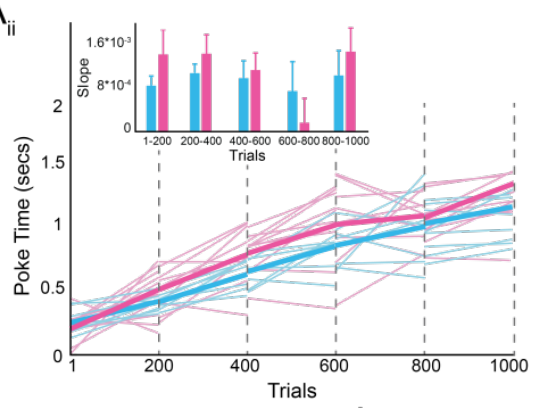

$\mathrm{A}_{\mathrm{iii}}$

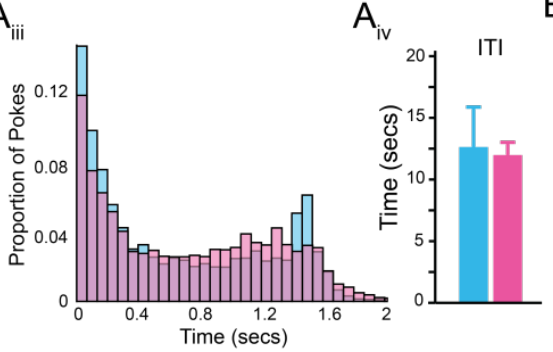

$\mathrm{B}_{\mathrm{i}}$

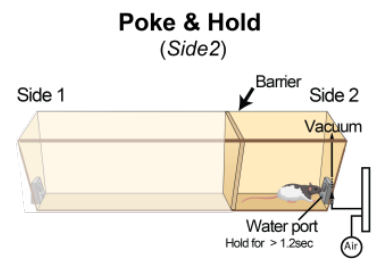

$\mathrm{B}_{\mathrm{ii}}$

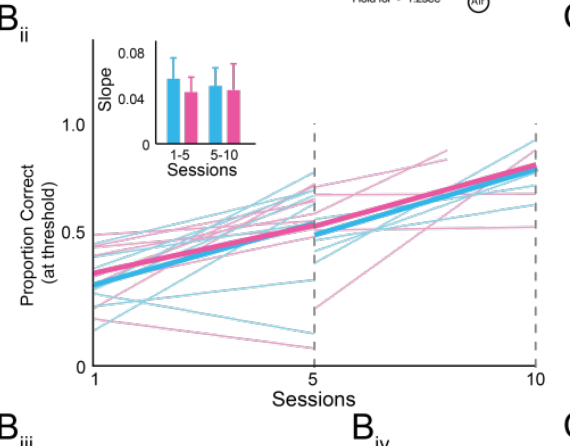

C

$\mathrm{C}_{\mathrm{ii}}$
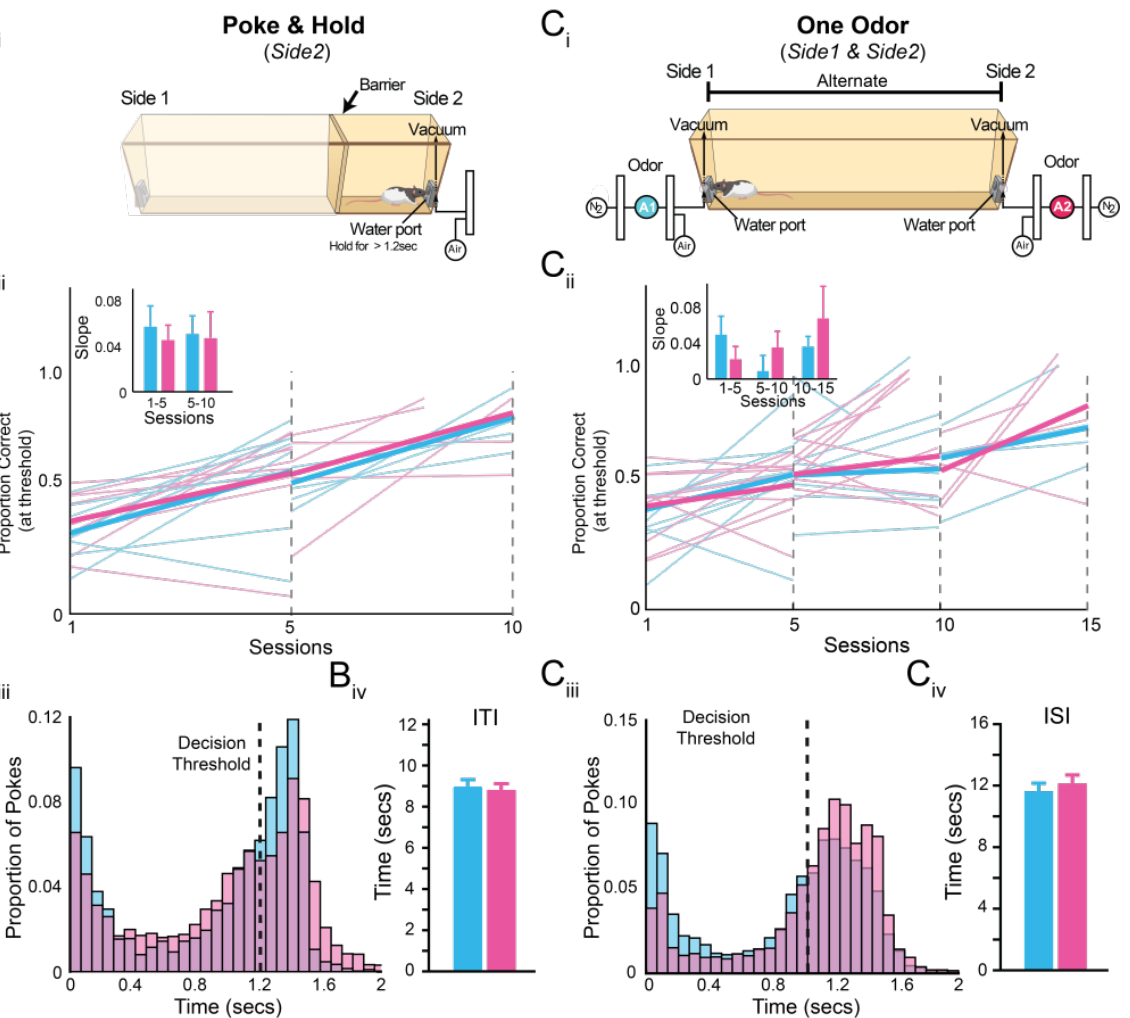

Figure 2. Males and Females Do Not Differ in Poking Behaviors

$\left(A_{i}\right)$ Rats were trained to hold their nose in the nose-port on side 1 for $1.2 \mathrm{~s}$.

$\left(A_{i i}\right)$ Males and females show no significant differences in the rate of learning across trials.

( $\left.A_{\text {iii }}\right)$ Males and females poke times were relatively similar.

$\left(A_{i v}\right)$ Inter-trial-interval (ITI) was not significantly different between males and females

$\left(B_{i}\right)$ Rats were trained to hold their nose in the nose-port on side 2 for $1.2 \mathrm{~s}$.

$\left(B_{i i}\right)$ No significant differences found between males and females in the rate of learning across sessions.

( $B_{\text {iii }}$ Males and females show subtle shifts in behavior where males show a tendency to have short poke times. Both males and females had increased number of pokes at the decision threshold (1.2 s).

$\left(B_{i v}\right)$ ITI between sexes were not significantly different.

$\left(\mathrm{C}_{\mathrm{i}}\right)$ Rats were introduced to one odor on either side of the maze and were trained to alternate holding their nose in the pose-port on both sides of the maze for $1 \mathrm{~s}$.

$\left(\mathrm{C}_{\mathrm{ii}}\right)$ No significant differences found between males and females in the rate of learning across sessions.

( $\left.\mathrm{C}_{\text {iii }}\right)$ Male and female rats show subtle shifts in behavior where males show a tendency to have shorter poke times.

$\left(\mathrm{C}_{\text {iv }}\right.$ ISI between males and females was not significantly different.

All data are represented as mean \pm SEM. ${ }^{*} p<0.05 ;{ }^{* *} p<0.01 ;{ }^{* * *} p<0.001$ 
bioRxiv preprint doi: https://doi.org/10.1101/2021.10.29.466512; this version posted November 1, 2021. The copyright holder for this preprint (which was not certified by peer review) is the author/funder, who has granted bioRxiv a license to display the preprint in perpetuity. It is made available under aCC-BY-NC-ND 4.0 International license.

SEX AND ESTROUS IN SEQUENCE MEMORY 22

$A_{i}$
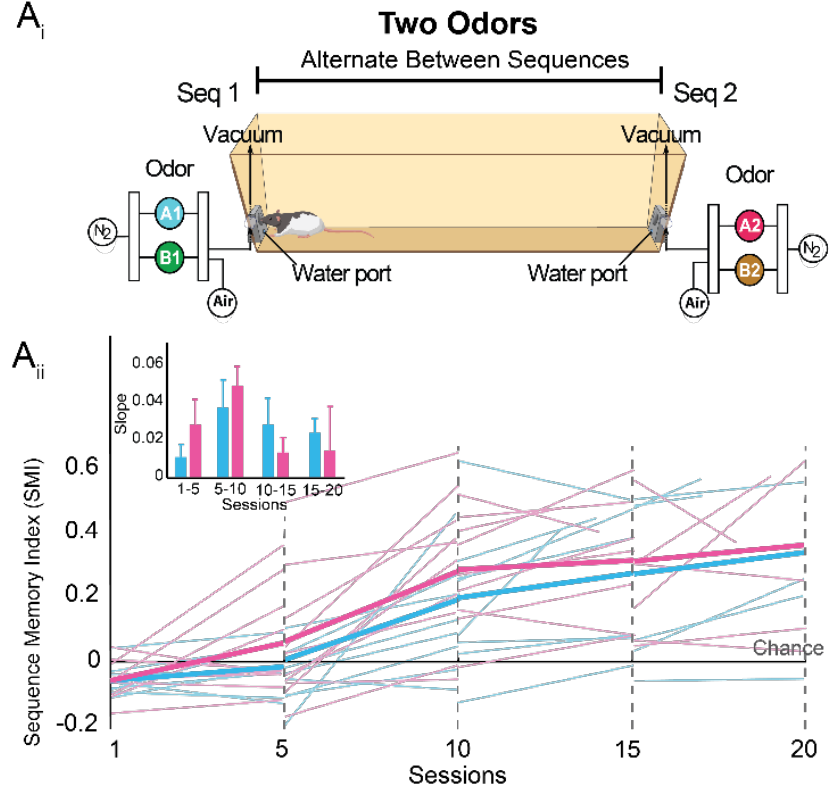

$A_{\text {iii }}$

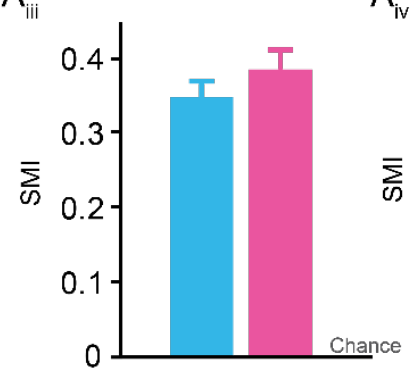

$\mathrm{A}_{\mathrm{iv}}$

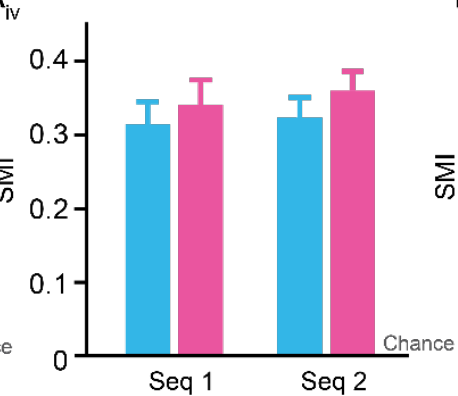

$B_{i}$
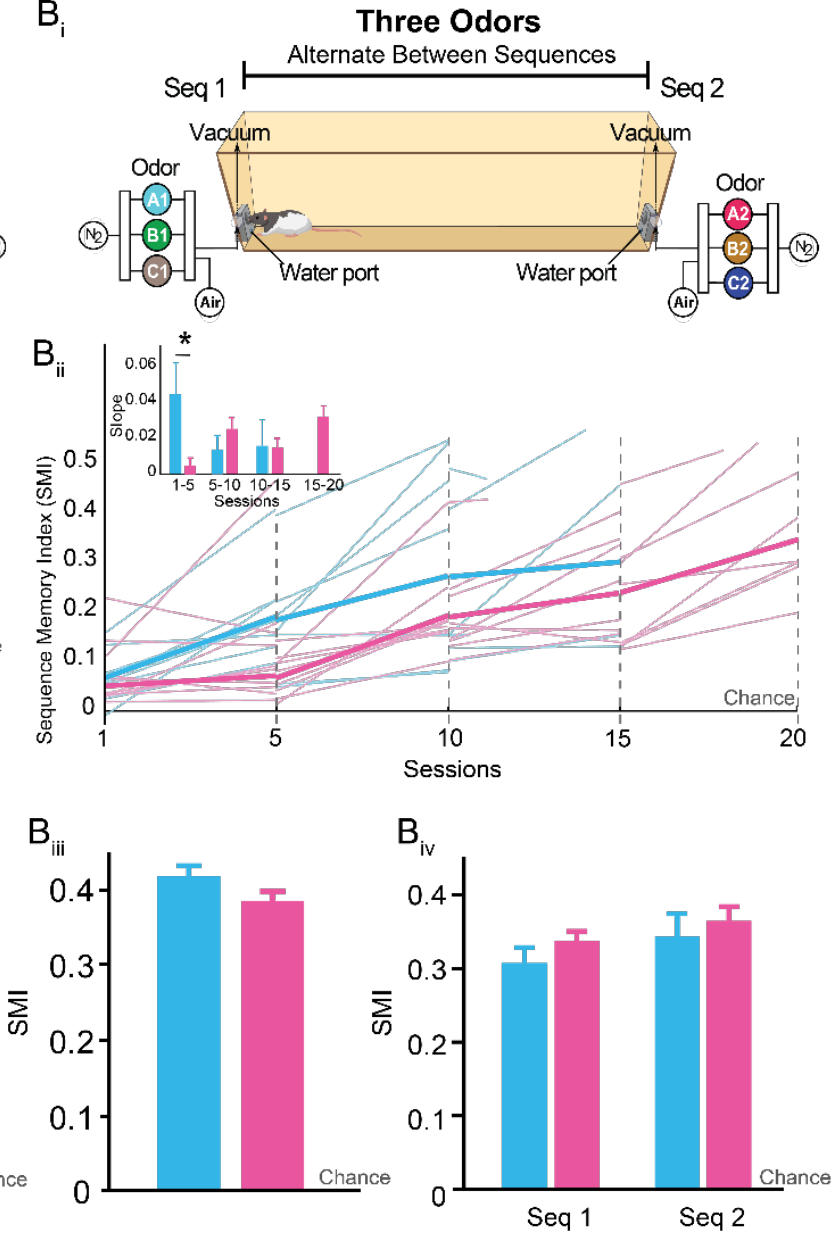

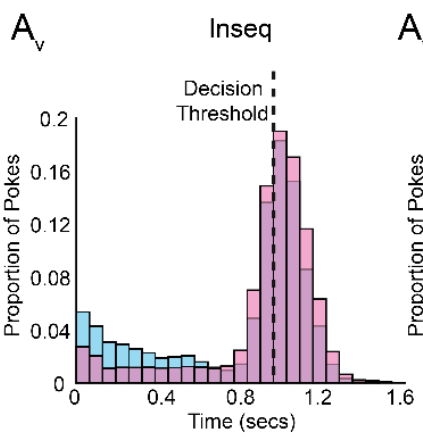

$A_{\text {vii }}$

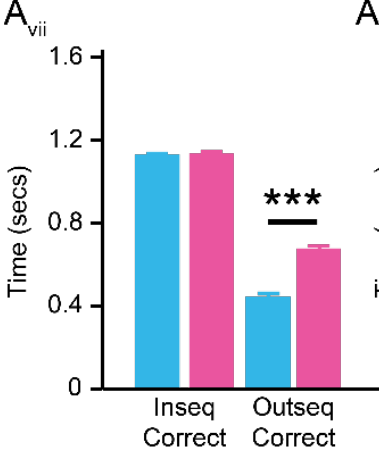

$A_{\text {viii }}$
$\mathrm{A}_{\mathrm{vi}}$
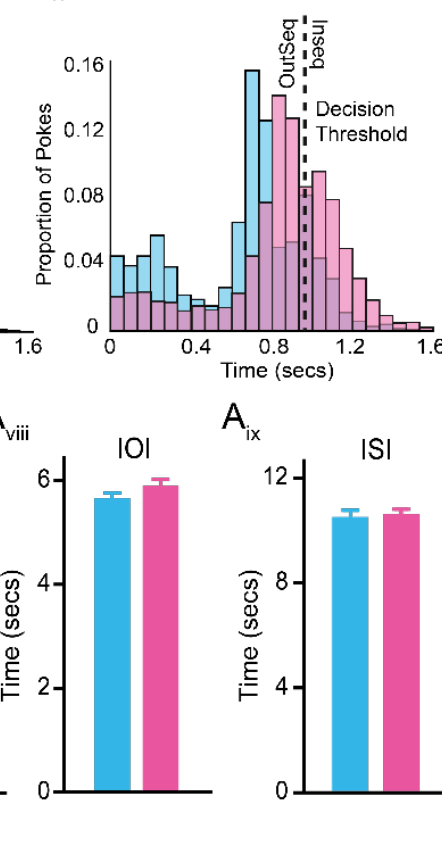

$A_{\text {ix }}$ ix $\quad|S|$

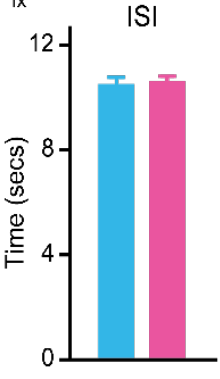

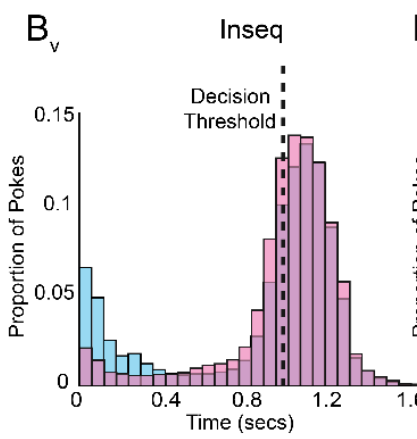

$\mathrm{B}_{\mathrm{vii}}$

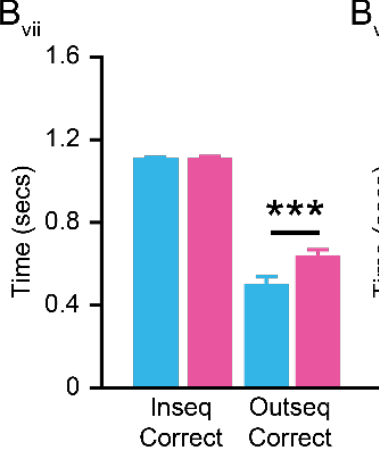

$\mathrm{B}_{\mathrm{vi}} \quad$ Outseq

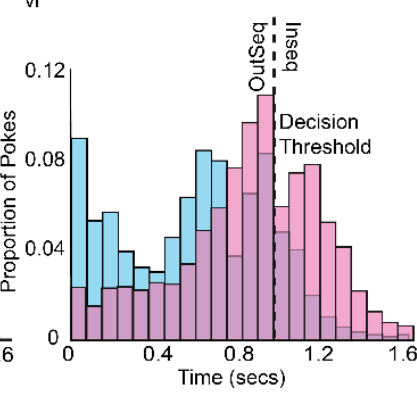

$\mathrm{B}_{\text {viii }}$

Figure 3. Learning Differs Between Males and Females

$\left(A_{i}\right)$ A second odor was added to either side of the maze (Seq1: $A_{1} B_{1}$ and Seq2: $A_{2} B_{2}$ ). 
$61 \quad\left(A_{i i}\right)$ Performance did not differ between males and females in the rate of learning across sessions.

62 ( $\left.\mathrm{A}_{\mathrm{iii}}\right) \mathrm{SMI}$ was not significantly different between males and females.

$63\left(A_{i v}\right)$ SMI was not significantly different between sequence 1 and sequence 2

$64\left(A_{v}\right)$ Males and females poke times were relatively similar.

$65\left(A_{v i}\right)$ Female nose-poke times show an increase in longer hold times while male poke times distribute 66 between short and long poke times

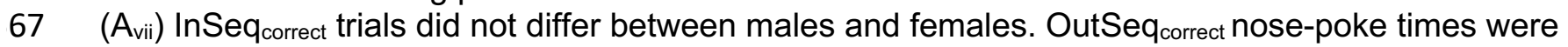
68 significantly different between males and females.

$69\left(A_{\text {viii }}\right)$ Inter-odor-interval (IOI) was not significantly different between sexes

$\left(A_{i x}\right)$ Inter-sequence-interval (ISI) was not significantly different between males and females

$\left(B_{i}\right) A$ third odor was added to both sides of the maze (Seq1: $A_{1} B_{1} C_{1}$ and Seq2: $\left.A_{2} B_{2} C_{2}\right)$.

$\left(B_{i i}\right)$ Learning rate between males and females was statistically significant during the first five sessions. As sessions continued, males and females no longer differed.

$\left(B_{i i i}\right) S M I$ was not significantly different between males and females.

$\left(B_{i v}\right)$ SMI was not significantly different between sequence 1 and sequence 2 .

$\left(B_{v}\right)$ Male and female poke times for InSeq trials were relatively similar. Males have an increase in shorter poke times.

$\left(B_{\mathrm{vi}}\right)$ Males show an increase number of short pokes for OutSeq trials while females showed longer hold times.

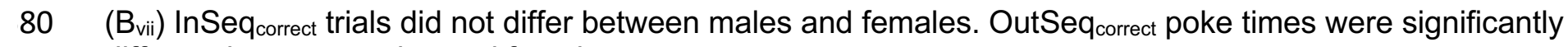
81 different between males and females.

$82 \quad\left(\mathrm{~B}_{\text {viii }}\right)$ IOI did not differ between males and females

$83\left(\mathrm{~B}_{\mathrm{ix}}\right)$ ISI did not differ between males and females. 
A
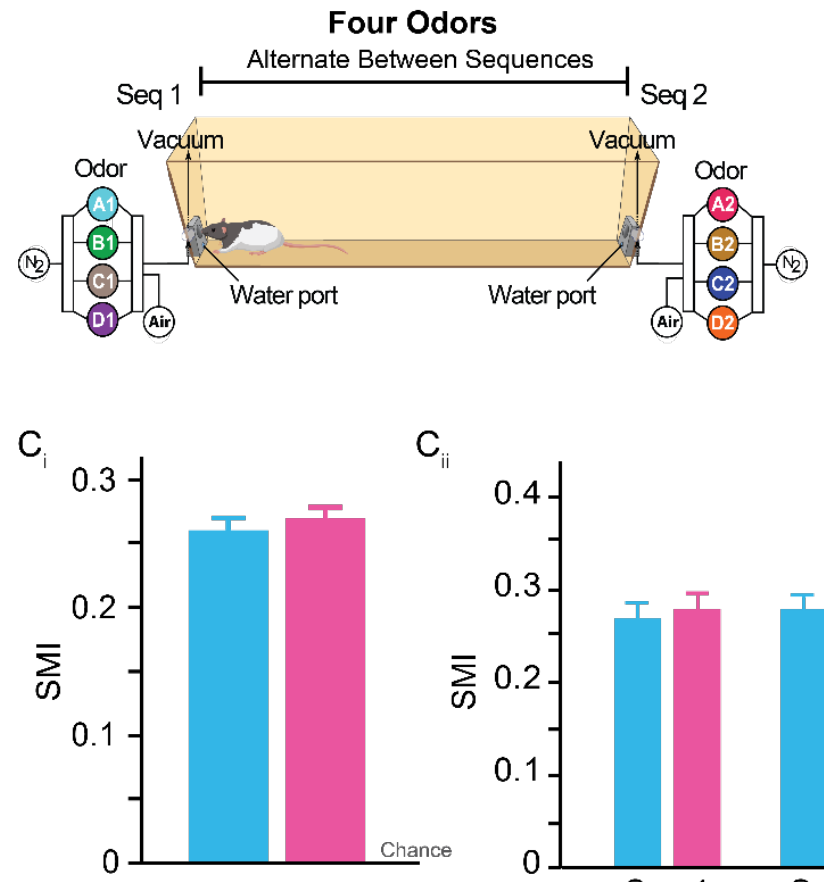

$F_{i}$

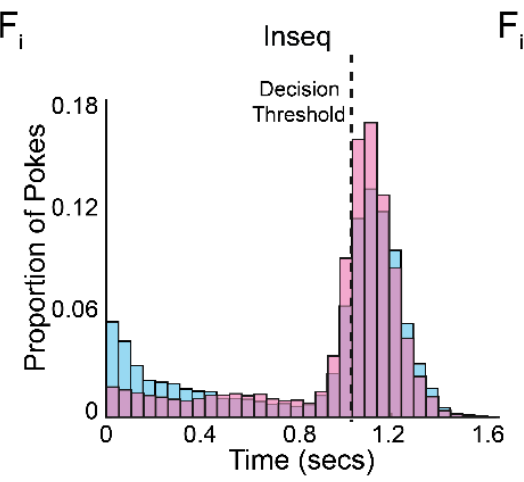

86 (Seq1: $A_{1} B_{1} C_{1} D_{1}$ and Seq2: $A_{2} B_{2} C_{2} D_{2}$ ). compared to females. difference between sexes.
B

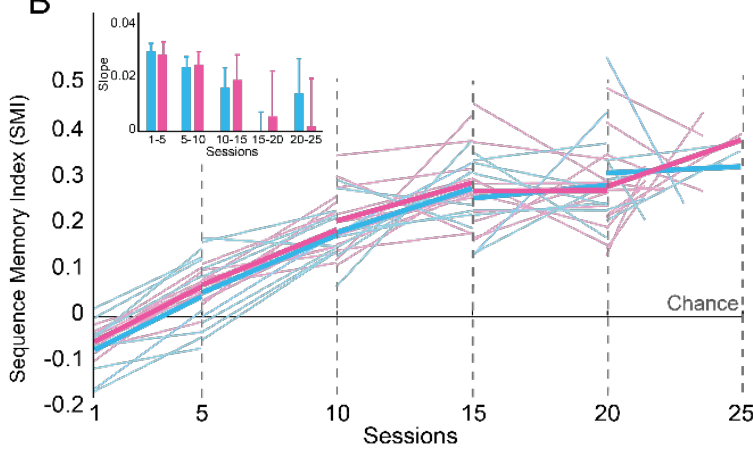

12

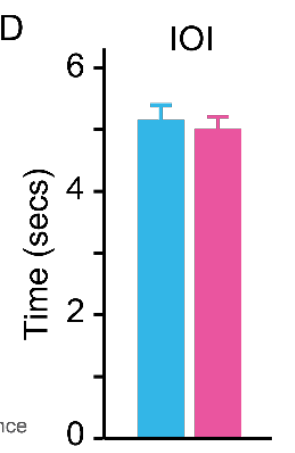

E $10+1 S I$

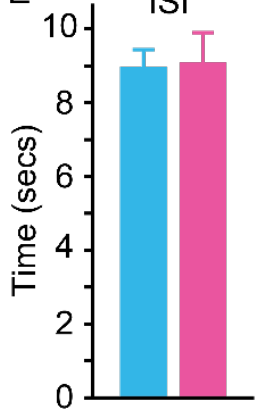

10

Figure 4. Males and Females Perform Similarly on the Sequence Memory Task

(A)Rats were trained train in the final stage of the sequence memory task with four odors in each sequence

(B) Males and females showed no significant differences in the rate of learning across sessions.

$\left(C_{i}\right) S M I$ was not significantly different between males and females.

$\left(\mathrm{C}_{\mathrm{ii}}\right) \mathrm{SMI}$ did not show any interaction effects between sequence 1 and sequence 2 and sex.

(D) IOI was not statistically different between males and females.

(E) ISI was not statistically different between males and females.

$\left(F_{i}\right)$ Male and female poke distributions are relatively similar for InSeq trials

$\left(F_{i i}\right)$ OutSeq poke distributions show subtle changes with males demonstrating an increase in shorter pokes

(G) InSeq correct $_{\text {trials did not differ between males and females. OutSeq }}$ correct trials showed a significant

All data are represented as mean \pm SEM. ${ }^{*} p<0.05 ;{ }^{* *} p<0.01 ;{ }^{* *} p<0.001$ 
A $\quad \mid \frac{10-12 \mathrm{H}}{\mid}$ Ovulation

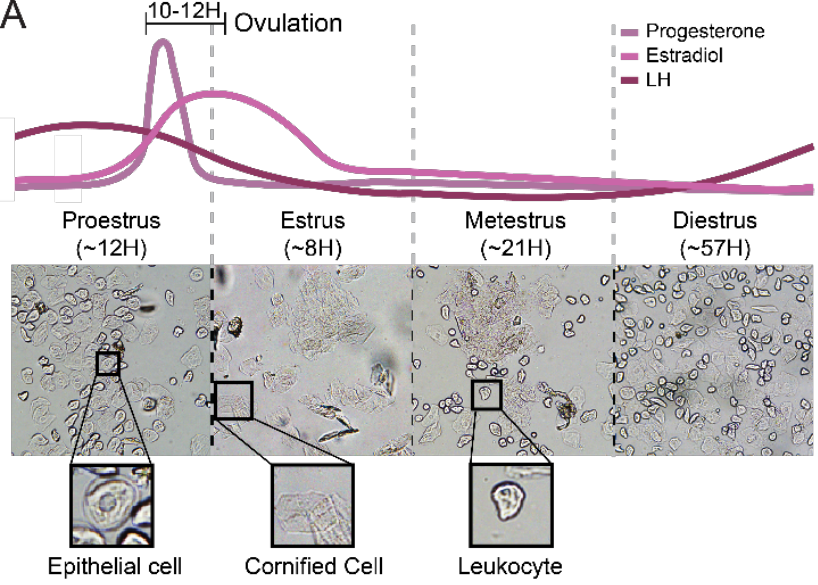

C

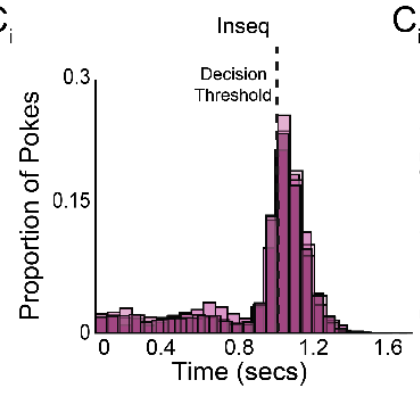

$\mathrm{C}_{\mathrm{ii}} \quad$ Outseq
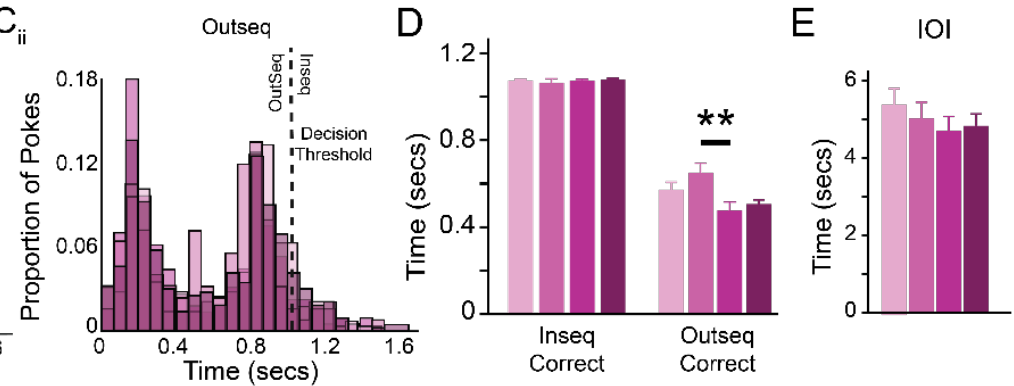

\section{Figure 5. Estrous Cycle Does Not Influence Sequence Memory}

(A)Rat ovulation hormone level fluctuations are shown for each stage as well as the amount of time each cell types (adapted from Goldman et al., 2007).

$\left(B_{i}\right)$ SMI was not different between estrous cycle phases.

$\left(B_{i i}\right) S M I$ for sequence 1 and sequence 2 showed no significant differences for the estrous cycle phases

$\left(C_{i}\right)$ InSeq poke distributions were relatively similar between estrous cycle phases. short pokes.

(D) InSeq correct $_{\text {trials did not differ between estrous cycle phases. OutSeq }}$ correct poke times were significantly different between estrus and metestrus.

(E) IOI was not statistically different between estrous cycle phases.

(F) ISI was not statistically different between estrous cycle phases.

All data are represented as mean \pm SEM. ${ }^{*} p<0.05 ;{ }^{* *} p<0.01 ;{ }^{* * *} p<0.001$
$\mathrm{E}$

$\mathrm{B}_{\mathrm{ii}}$

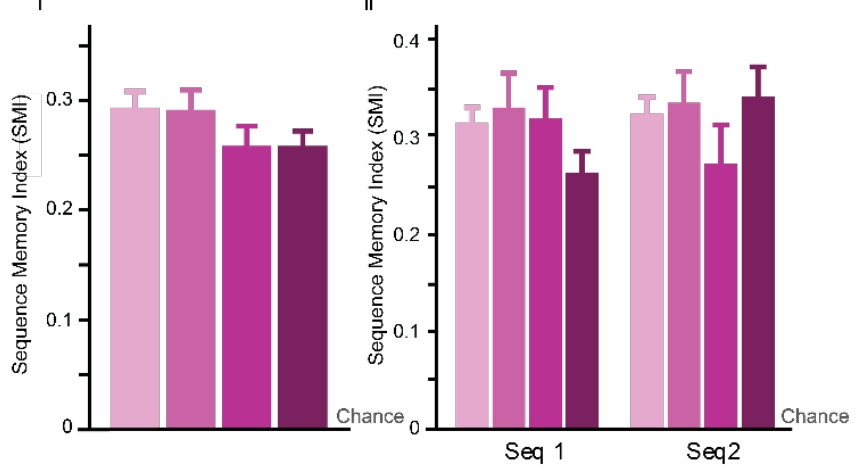

$\mathrm{F}$

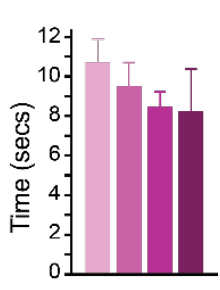
stage lasts. Lavage sample images of each phase of the estrous cycle with zoomed images of the primary

$\left(\mathrm{C}_{\text {iii }}\right)$ OutSeq poke distributions showed subtle behavioral differences with estrus showing slightly increased 
SEX AND ESTROUS IN SEQUENCE MEMORY 26

\section{REFRENCES}

Allen, L. M., Lesyshyn, R. A., O’Dell S. J., Allen, T. A., Fortin, N. J. (2020). The hippocampus, prefrontal cortex, and perirhinal cortex are critical to incidental order memory. Behavioural Brain Research, 379, 112215. https://doi.org/10.1016/j.bbr.2019.112215

Allen, T. A., Morris, A. M., Mattfeld, A. T., Stark, C. E. L., \& Fortin, N. J. (2014). A sequence of events model of episodic memory shows parallels in rats and humans. Hippocampus, 24(10), 1178-1188. https://doi.org/10.1002/hipo.22301

Allen, T. A., Morris, A. M., Stark, S. M., Fortin, N. J., \& Stark, C. E. L. (2015). Memory for sequences of events impaired in typical aging. Learning \& Memory, 22(3). https://doi.org/10.1101/lm.036301.114

Allen, T. A., Salz, D. M., McKenzie, S., \& Fortin, N. J. (2016). Nonspatial Sequence Coding in CA1 Neurons. The Journal of Neuroscience, 36(5). https://doi.org/10.1523/JNEUROSCI.2874-15.2016

Bannerman, D. M., Good, M. A., Butcher, S. P., Ramsay, M., \& Morris, R. G. M. (1995). Distinct components of spatial learning revealed by prior training and NMDA receptor blockade. Nature, 378(6553). https://doi.org/10.1038/378182a0

Barha, C. K., Dalton, G. L., \& Galea, L. A. (2010). Low Doses of 17a-Estradiol and 17ß-Estradiol Facilitate,

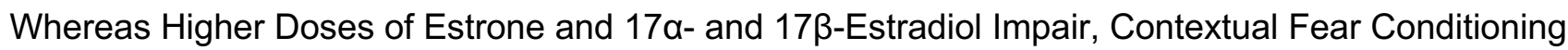
in Adult Female Rats. Neuropsychopharmacology, 35(2). https://doi.org/10.1038/npp.2009.161

Berry, B., McMahan, R., \& Gallagher, M. (1997). Spatial learning and memory at defined points of the estrous cycle: Effects on performance of a hippocampal-dependent task. Behavioral Neuroscience, 111(2). https://doi.org/10.1037/0735-7044.111.2.267

Bettis, T., \& Jacobs, L. F. (2012). Sex differences in object recognition are modulated by object similarity. Behavioural Brain Research, 233(2), 288-292. https://doi.org/10.1016/j.bbr.2012.04.028

Blanchard, R. J., Carol, D., \& Blanchard, V. (1968). Passive avoidance: A variety of fear conditioning? In Psych on. Sci (Vol. 13). DOI:10.3758/BF03342386

Bucci, D. J., Chiba, A. A., \& Gallagher, M. (1995). Spatial learning in male and female Long-Evans rats. Behavioral Neuroscience, 135(1). https://doi.org/10.1037/bne0000437 
52

53

54

55

56

57

58

59

60

61

62

63

64

65

66

67

68

69

70

71

72

73

74

75

76

Bucci, D. J., Hopkins, M. E., Nunez, A. A., Breedlove, S. M., Sisk, C. L., \& Nigg, J. T. (2008). Effects of sex hormones on associative learning in spontaneously hypertensive rats. Physiology \& Behavior, 93(3). https://doi.org/10.1016/j.physbeh.2007.11.005

Chai, X. J., \& Jacobs, L. F. (2010). Effects of cue types on sex differences in human spatial memory. Behavioural Brain Research, 208(2), 336-342. https://doi.org/10.1016/j.bbr.2009.11.039

Chesler, E. J., \& Juraska, J. M. (2000). Acute Administration of Estrogen and Progesterone Impairs the Acquisition of the Spatial Morris Water Maze in Ovariectomized Rats. Hormones and Behavior, 38(4). https://doi.org/10.1006/hbeh.2000.1626

Cimadevilla, J. M., Fenton, A. A., \& Bures, J. (2000). Continuous place avoidance task reveals differences in spatial navigation in male and female rats. Behavioural Brain Research, 107(1-2). https://doi.org/10.1016/S0166-4328(99)00128-X

Conrad, C. D., Jackson, J. L., Wieczorek, L., Baran, S. E., Harman, J. S., Wright, R. L., \& Korol, D. L. (2004). Acute stress impairs spatial memory in male but not female rats: influence of estrous cycle. Pharmacology Biochemistry and Behavior, 78(3). https://doi.org/10.1016/j.pbb.2004.04.025

Davis, D. M., Jacobson, T. K., Aliakbari, S., \& Mizumori, S. J. Y. (2005). Differential effects of estrogen on hippocampal- and striatal-dependent learning. Neurobiology of Learning and Memory, 84(2), 132137. https://doi.org/10.1016/j.nlm.2005.06.004

Dohanich, G. (2002). Gonadal Steroids, Learning, and Memory. In Hormones, Brain and Behavior. Elsevier. https://doi.org/10.1016/B978-012532104-4/50024-X

Eichenbaum, H., \& Fortin, N. J. (2005). Bridging the gap between brain and behavior: cognitive and neural mechanisms of episodic memory. Journal of the Experimental Analysis of Behavior, 84(3). https://doi.org/10.1901/jeab.2005.80-04

Frick, K. M., \& Berger-Sweeney, J. (2001). Spatial reference memory and neocortical neurochemistry vary with the estrous cycle in C57BL/6 mice. Behavioral Neuroscience, 115(1). https://doi.org/10.1037/0735-7044.115.1.229 
Frick, K. M., Burlingame, L. A., Arters, J. A., \& Berger-Sweeney, J. (1999). Reference memory, anxiety and estrous cyclicity in c57bl/6nia mice are affected by age and sex. www.elsevier.com/locate/neuroscience

Frye, C. A. (1995). Estrus-associated decrements in a water maze task are limited to acquisition. Physiology \& Behavior, 57(1). https://doi.org/10.1016/0031-9384(94)00197-D

Frye, C. A., Duffy, C. K., \& Walf, A. A. (2007). Estrogens and progestins enhance spatial learning of intact and ovariectomized rats in the object placement task. Neurobiology of Learning and Memory, 88(2). https://doi.org/10.1016/j.nlm.2007.04.003

Goldman, J. M., Murr, A. S., \& Cooper, R. L. (2007). The rodent estrous cycle: Characterization of vaginal cytology and its utility in toxicological studies. In Birth Defects Research Part B - Developmental and Reproductive Toxicology (Vol. 80, Issue 2, pp. 84-97). https://doi.org/10.1002/bdrb.20106

Gruene, T. M., Flick, K., Stefano, A., Shea, S. D., \& Shansky, R. M. (2015). Sexually divergent expression of active and passive conditioned fear responses in rats. ELife, 4. https://doi.org/10.7554/eLife.11352

Hamson, D. K., Roes, M. M., \& Galea, L. A. M. (2016). Sex Hormones and Cognition: Neuroendocrine Influences on Memory and Learning. In Comprehensive Physiology (Vol. 6, Issue 3, pp. 1295-1337). https://doi.org/10.1002/cphy.c150031

Healy, S. D., Braham, S. R., \& Braithwaite, V. A. (1999). Spatial working memory in rats: no differences between the sexes. Proceedings of the Royal Society of London. Series B: Biological Sciences, 266(1435). https://doi.org/10.1098/rspb.1999.0923

Jayachandran, M., Linley, S. B., Schlecht, M., Mahler, S. v., Vertes, R. P., \& Allen, T. A. (2019). Prefrontal Pathways Provide Top-Down Control of Memory for Sequences of Events. Cell Reports, 28(3), 640654.e6. https://doi.org/10.1016/j.celrep.2019.06.053

Jonasson, Z. (2005). Meta-analysis of sex differences in rodent models of learning and memory: a review of behavioral and biological data. Neuroscience \& Biobehavioral Reviews, 28(8). 
02

03

04

05

06

07

08

09

10

11

12

13

14

15

16

17

18

19

20

21

22

23

24

25

26

27

Kessler, R. C., Berglund, P., Demler, O., Jin, R., Merikangas, K. R., \& Walters, E. E. (2005). Lifetime Prevalence and Age-of-Onset Distributions of DSM-IV Disorders in the National Comorbidity Survey Replication. Archives of General Psychiatry, 62(6). https://doi.org/10.1001/archpsyc.62.6.593

Korol, D. L. (2004). Role of estrogen in balancing contributions from multiple memory systems. Neurobiology of Learning and Memory, 82(3). https://doi.org/10.1016/j.nlm.2004.07.006

Korol, D. L., \& Kolo, L. L. (2002). Estrogen-induced changes in place and response learning in young adult female rats. Behavioral Neuroscience, 116(3). https://doi.org/10.1037/0735-7044.116.3.411

Koss, W. A., \& Frick, K. M. (2017). Sex differences in hippocampal function. In Journal of Neuroscience Research (Vol. 95, Issues 1-2, pp. 539-562). John Wiley and Sons Inc. https://doi.org/10.1002/jnr.23864

Lamberty, Y., \& Gower, A. J. (1988). Investigation into sex-related differences in locomotor activity, place learning and passive avoidance responding in NMRI mice. Physiology \& Behavior, 44(6). https://doi.org/10.1016/0031-9384(88)90063-7

Launer, J. (1999). Narrative based medicine: A narrative approach to mental health in general practice. BMJ, 318(7176). https://doi.org/10.1136/bmj.318.7176.117

Levy, L. J., Astur, R. S., \& Frick, K. M. (2005). Men and Women Differ in Object Memory but Not Performance of a Virtual Radial Maze. Behavioral Neuroscience, 119(4). https://doi.org/10.1037/0735-7044.119.4.853

Luine, V., Gomez, J., Beck, K., \& Bowman, R. (2017). Sex differences in chronic stress effects on cognition in rodents. Pharmacology Biochemistry and Behavior, 152, 13-19. https://doi.org/10.1016/j.pbb.2016.08.005

McCarthy, M. M., \& Konkle, A. T. M. (2005). When is a sex difference not a sex difference? Frontiers in Neuroendocrinology, 26(2). https://doi.org/10.1016/j.yfrne.2005.06.001

McEwen, B. S., \& Milner, T. A. (2017). Understanding the broad influence of sex hormones and sex differences in the brain. Journal of Neuroscience Research, 95(1-2). https://doi.org/10.1002/jnr.23809 
Nowak, N. T., Diamond, M. P., Land, S. J., \& Moffat, S. D. (2014). Contributions of sex, testosterone, and androgen receptor CAG repeat number to virtual Morris water maze performance. Psychoneuroendocrinology, 41. https://doi.org/10.1016/j.psyneuen.2013.12.003

Paris, J. J., \& Frye, C. A. (2008). Estrous cycle, pregnancy, and parity enhance performance of rats in object recognition or object placement tasks. Reproduction, 136(1). https://doi.org/10.1530/REP-07-0512

Perrot-Sinal, T. S., Kostenuik, M. A., Ossenkopp, K.-P., \& Kavaliers, M. (1996). Sex differences in performance in the Morris water maze and the effects of initial nonstationary hidden platform training. Behavioral Neuroscience, 110(6). https://doi.org/10.1037/0735-7044.110.6.1309

Pigott, T. A. (2003). Anxiety disorders in women. Psychiatric Clinics of North America, 26(3). https://doi.org/10.1016/S0193-953X(03)00040-6

Pompili, A., Tomaz, C., Arnone, B., Tavares, M. C., \& Gasbarri, A. (2010). Working and reference memory across the estrous cycle of rat: A long-term study in gonadally intact females. Behavioural Brain Research, 213(1). https://doi.org/10.1016/j.bbr.2010.04.018

Prendergast, B. J., Onishi, K. G., \& Zucker, I. (2014). Female mice liberated for inclusion in neuroscience and biomedical research. Neuroscience \& Biobehavioral Reviews, 40. https://doi.org/10.1016/j.neubiorev.2014.01.001

Reeders, P. C., Hamm, A. G., Allen, T. A., \& Mattfeld, A. T. (2021). Medial prefrontal cortex and hippocampal activity differentially contribute to ordinal and temporal context retrieval during sequence memory. Learning \& Memory, 28(4). https://doi.org/10.1101/lm.052365.120

Roof, R. L., Zhang, Q., Glasier, M. M., \& Stein, D. G. (1993). Gender-specific impairment on Morris water maze task after entorhinal cortex lesion. Behavioural Brain Research, 57(1). https://doi.org/10.1016/0166-4328(93)90060-4

Roof, R., \& Stein, D. (1999). Gender differences in Morris water maze performance depend on task parameters. Physiology \& Behavior, 68(1-2). https://doi.org/10.1016/S0031-9384(99)00162-6

Saucier, D., \& Cain, D. P. (1995). Spatial learning without NMDA receptor-dependent long-term potentiation. Nature, 378(6553). https://doi.org/10.1038/378186a0 
54 Saucier, D. M., Shultz, S. R., Keller, A. J., Cook, C. M., \& Binsted, G. (2008). Sex differences in object

55 location memory and spatial navigation in Long-Evans rats. Animal Cognition, 11(1), 129-137. https://doi.org/10.1007/s10071-007-0096-1

Schmidt, B., Jacobson, T. K., \& Markus, E. (2009). Hippocampal and striatal dependent navigation: Sex differences are limited to acquisition. Hormones and Behavior, 56(2), 199-205. https://doi.org/10.1016/j.yhbeh.2009.04.004

Seymoure, P., Dou, H., \& Juraska, J. M. (1996). Sex differences in radial maze performance: Influence of rearing environment and room cues. In Psychobiology (Vol. 24, Issue 1). https://doi.org/10.3758/BF03331950

Sherry, D. F., \& Hampson, E. (1997). Evolution and the hormonal control of sexually-dimorphic spatial abilities in humans. Trends in Cognitive Sciences, 1(2). https://doi.org/10.1016/S13646613(97)01015-2

Sokal, Robert. R., \& Rohlf, J. F. (1995). Biometry: The principles and practice of statistics in Biological Research (Vol. 2nd). Freeman.

Spritzer, M. D., Gill, M., Weinberg, A., \& Galea, L. A. M. (2008). Castration Differentially Affects Spatial Working and Reference Memory in Male Rats. Archives of Sexual Behavior, 37(1). https://doi.org/10.1007/s10508-007-9264-2

Stackman, R. W., Blasberg, M. E., Langan, C. J., \& Clark, A. S. (1997). Stability of Spatial Working Memory across the Estrous Cycle of Long-Evans Rats. Neurobiology of Learning and Memory, 67(2). https://doi.org/10.1006/nlme.1996.3753

Steimer, T., \& Driscoll, P. (2005). Inter-individual vs line/strain differences in psychogenetically selected Roman High-(RHA) and Low-(RLA) Avoidance rats: Neuroendocrine and behavioural aspects. In Neuroscience and Biobehavioral Reviews (Vol. 29, Issue 1 SPEC. ISS., pp. 99-112). Elsevier Ltd. https://doi.org/10.1016/j.neubiorev.2004.07.002

Sutcliffe, J. S., Marshall, K. M., \& Neill, J. C. (2007). Influence of gender on working and spatial memory in the novel object recognition task in the rat. Behavioural Brain Research, 177(1). https://doi.org/10.1016/j.bbr.2006.10.029 
81 Tulving, E. (2002). Episodic Memory: From Mind to Brain. Annual Review of Psychology, 53(1).

82

83

84

85

86

87

88

89

90

91

92

93

94

95

96

97

98

99

00

01

02

03

04

05

06 https://doi.org/10.1146/annurev.psych.53.100901.135114

van Goethem, N. P., Rutten, K., van der Staay, F. J., Jans, L. A. W., Akkerman, S., Steinbusch, H. W. M., Blokland, A., van't Klooster, J., \& Prickaerts, J. (2012). Object recognition testing: Rodent species, strains, housing conditions, and estrous cycle. Behavioural Brain Research, 232(2). https://doi.org/10.1016/j.bbr.2012.03.023

Veng, L. M., Granholm, A.-C., \& Rose, G. M. (2003). Age-related sex differences in spatial learning and basal forebrain cholinergic neurons in F344 rats. Physiology \& Behavior, 80(1). https://doi.org/10.1016/S0031-9384(03)00219-1

Walf, A. A., \& Frye, C. A. (2006). A Review and Update of Mechanisms of Estrogen in the Hippocampus and Amygdala for Anxiety and Depression Behavior. Neuropsychopharmacology, 31(6). https://doi.org/10.1038/sj.npp.1301067

Warren, S. G., \& Juraska, J. M. (1997). Spatial and nonspatial learning across the rat estrous cycle. Behavioral Neuroscience, 111(2). https://doi.org/10.1037/0735-7044.111.2.259

Williams, C. L., Barnett, A. M., \& Meck, W. H. (1990). Organizational effects of early gonadal secretions on sexual differentiation in spatial memory. Behavioral Neuroscience, 104(1). https://doi.org/10.1037/0735-7044.104.1.84

Woolley, D. G., Vermaercke, B., de Beeck, H. O., Wagemans, J., Gantois, I., D’Hooge, R., Swinnen, S. P., \& Wenderoth, N. (2010). Sex differences in human virtual water maze performance: Novel measures reveal the relative contribution of directional responding and spatial knowledge. Behavioural Brain Research, 208(2), 408-414. https://doi.org/10.1016/j.bbr.2009.12.019

Zurkovsky, L., Brown, S. L., Boyd, S. E., Fell, J. A., \& Korol, D. L. (2007). Estrogen modulates learning in female rats by acting directly at distinct memory systems. Neuroscience, 144(1), 26-37. https://doi.org/10.1016/j.neuroscience.2006.09.002 